\title{
AUTOMATIC ORIENTATION AND MOSAICKING OF ARCHIVED AERIAL PHOTOGRAPHY USING STRUCTURE FROM MOTION
}

\author{
J. A. Gonçalves \\ Faculdade Ciências - Universidade Porto, \\ Rua Campo Alegre 4169-007 Porto, Portugal - jagoncal@fc.up.pt
}

\section{KEY WORDS:}

Archived aerial photos, Structure from Motion, Bundle adjustment, Auto-calibration, DSM, Orthomosaics

\begin{abstract}
:
Aerial photography has been acquired regularly for topographic mapping since the decade of 1930. In Portugal there are several archives of aerial photos in national mapping institutes, as well as in local authorities, containing a total of nearly one hundred thousand photographs, mainly from the 1940s, 1950s and some from 1930s. These data sets provide important information about the evolution of the territory, for environment and agricultural studies, land planning, and many other examples. There is an interest in making these aerial coverages available in the form of orthorectified mosaics for integration in a GIS.

The orthorectification of old photographs may pose several difficulties. Required data about the camera and lens system used, such as the focal distance, fiducial marks coordinates or distortion parameters may not be available, making it difficult to process these data in conventional photogrammetric software.

This paper describes an essentially automatic methodology for orientation, orthorectification and mosaic composition of blocks of old aerial photographs, using Agisoft Photoscan structure from motion software. The operation sequence is similar to the processing of UAV imagery. The method was applied to photographs from 1947 and 1958, provided by the Portuguese Army Geographic Institute. The orientation was done with GCPs collected from recent orthophototos and topographic maps. This may be a difficult task, especially in urban areas that went through many changes. Residuals were in general below 1 meter. The agreement of the orthomosaics with recent orthophotos and GIS vector data was in general very good. The process is relatively fast and automatic, and can be considered in the processing of full coverages of old aerial photographs.
\end{abstract}

\section{INTRODUCTION}

In Portugal there are several archives of aerial photos in national mapping institutes, as well as in local authorities, containing more than one hundred thousand photographs, from the decades 1930, 1940 and 1950s. These data sets provide important information about the evolution of the territory, for environment and agricultural studies, land planning, legal boundaries disputes, and many other examples. There is an interest in making these aerial coverages available in the form of orthorectified mosaics for integration in a GIS.

Aerial photography has been acquired regularly for topographic mapping since the decade of 1930. The Portuguese Army started the production of topographic maps at scale 1:25,000, by stereoscopic restitution, in this period. A local company Sociedade Portuguesa de Levantamentos Aéreos Ltd. (SPLAL) - made the aerial coverages, resulting in 40,000 photos that are now property of the Army Geographic Institute (IGeoE Instituto Geográfico do Exército). The camera used was a Zeiss RMK S1818, with film size of $18 \mathrm{~cm}$ and a $204 \mathrm{~mm}$ lens (Redweik et al., 2010).

Other full coverages were also made. After the second world war, in 1947, the Royal Air Force made a uniform coverage of all Portugal, in scale 1:30,000, in a total of 10,000 photos, also property of IGeoE. In 1958 the United Sates Air Force made a full coverage of Portugal and Spain, resulting in some 12,000 photos for all Portugal. These two coverages (RAF47 and USAF58) were made using cameras with film size 23 by $23 \mathrm{~cm}$ and $152 \mathrm{~mm}$ lens. Figure 1 shows photos of SPLAL and RAF47 flights.
These collections were analysed with great detail by Redweik et al. (2010). It became possible to know some characteristics of the cameras used, which are important for a photogrammetric processing of those images. Anyway, conventional photogrammetric treatment of these photographs, in order to produce orthophotos would be time consuming and relatively expensive. At least, up to this moment no significant works of orthorectifying old aerial photos have been done.
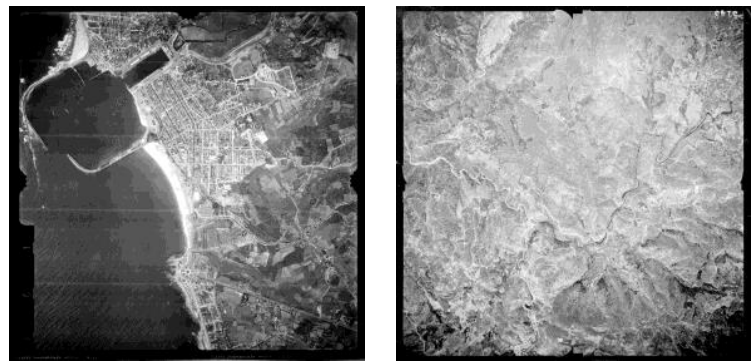

Figure 1 - Examples of photos from SPLAL and RAF flights, both from 1947.

The more common situation is that calibration certificates, or even basic knowledge of camera characteristics, are missing, making the use of those images even more difficult. In cases of flat terrain, simple bi-dimensional image georeferencing with polynomials may be used, as is the case of Alhaddad, et al. (2009) who describe a study of urban sprawl analysis using archived photographs. An alternative method, applicable in generic cases, is the Direct Linear Transformation (DLT) orientation method (Abdel-Aziz and Karara, 1972), which does not require camera information, but has the disadvantage of needing many ground control points. Ma and Buchwald (2012), 
for example, describe its use in small blocks of archived aerial photographs.

More efficient and largely automatic methods to orthorectify archived aerial phots are needed. Important recent developments in digital photogrammetry and computer vision algorithms can provide solutions for this task. This paper deals with a methodology based on those approaches, to orient and build orthomosaics of archived aerial photographs, similar to what is done with imagery acquired by unmanned aerial vehicles (UAV).

Many of these archived photos are already digitised by IGeoE, in a photogrammetric scanner, with a resolution of $0.021 \mathrm{~mm}$. Some of the SPLAL and USAF photos were tested in this work.

\section{PHOTOGRAMMETRIC PROCESSING OF UAV} IMAGERY

UAVs are now commonly used to acquire coverages of hundreds of aerial images, with compact cameras. Processing methodologies commonly used are largely automated, for example by commercial software packages such as Pix4D, Agisoft Photoscan and Trimble UAS Master, or open source, such as MicMac (Deseilligny and Clery, 2011). These methodologies, in part originated in computer vision, are designated as Structure from Motion (SfM), (Abdel-Wahab et al., 2012). This method inspired the solution proposed for archived aerial photos.

\subsection{UAV image processing with SfM}

UAV images are acquired by compact cameras for which only approximate parameters are known (e.g. nominal focal length) and can have radial distortions that are not calibrated. This situation is similar to what happens with old photos, for which calibration certificates are not available. UAVs are supported by navigation GPS receivers, which can provide approximate position of the camera projection centres. The processing steps are the following:

1. Apply algorithms similar to SIFT (Scale Invariant Feature Transform), (Lowe, 2004), which give many conjugate points between overlapping images.

2. Do a free bundle adjustment in order to obtain a relative orientation of all the images. This operation is called in the SfM approach, "Image alignment". With the navigation GPS positions an approximate georeferencing can be done.

3. If more accurate georeferencing is needed, ground control points (GCPs) can be provided, in order to do an aerial triangulation, incorporating an auto-calibration.

4. Dense matching algorithms are used to generate a dense point cloud, by multi view stereo matching. A regular grid digital surface model (DSM) is obtained from the point cloud.

5. Images are orthorectified (true ortho if the DSM is used) and a continuous mosaic is built.

This workflow is similar in several programs. The one used in this work to process the old photos was Agisoft Photoscan.

\subsection{Camera calibration}

The distortion model used is the Brown distortion model (Agisoft, 2015), which considers the following parameters to describe a camera:
$f \quad$ focal length,
$c_{x}, c_{y} \quad$ principal point coordinates,
$k_{1}, k_{2}, k_{3}$ radial distortion polynomial coefficients,
$p_{1}, p_{2} \quad$ tangential distortion coefficients.

There are also parameters to account for skew effect and for $x$ and $y$ different scales, which are treated as 2 focal distances.

The bundle adjustment considers an auto-calibration, in which the user chooses which of these parameters he wants to adjust. This process requires many and well distributed conjugate points, as well as control points.

\section{PROPOSED METHODOLOGY}

\subsection{Image preparation}

This software expects digital images, and the old photos are digitised, film based photos. In a standard photogrammetric treatment of digitised photos, an interior orientation, with fiducial marks, should be done. In this case it will be replaced by a resampling of all photos, such that all images have the same size and the same position with respect to the photographic coordinate system. That is done by an image registration, through an affine transformation.

Since in a general case, coordinates of fiducial marks will not be available, the chosen approach will be to take one of the images as a reference image and register all the others to it, using the fiducial marks. Figure 2 shows examples of fiducial marks of photos of the three flights referred before.

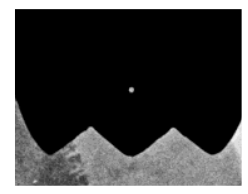

SPLAL

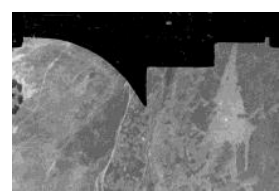

RAF47

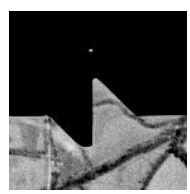

USAF58
Figure 2 - Examples of fiducial marks of photos from the SPLAL, RAF47 and USAF58 flights.

In a case where the fiducial marks are not clearly identified, reference points in the image frame can be used, provided that they are stable from image to image.

In the manual registration done in all tests, the residuals of the affine transformation with 4 fiducial marks were normally under 1 pixel. In future works involving the processing of large numbers of photos, some automation can be easily done, in a similar way to what is done in an automatic interior orientation, due to the repetitive pattern of the marks.

\subsection{Image orientation}

The following step is the image alignment, in which conjugate points are obtained automatically. Figure 3 shows conjugate points obtained between two overlapping images of the SPLAL flight. That is followed by the "image alignment". GCPs can be provided at this stage in order to orientate all the block. Figure 4 shows the result of image orientation, including the sparse point cloud and the GCPs.

The image orientation is optimized in a bundle adjustment with camera auto-calibration, where the user chooses the parameters to be considered. Statistics of the residuals found on the GCPs and the conjugate points are provided, and once they are small, 
the result can be accepted. Accuracy assessment can be done more easily over the final products.

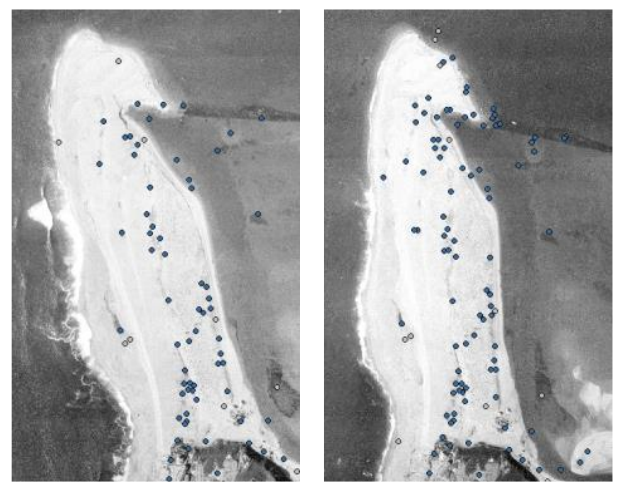

Figure 3 - Examples of conjugate points obtained between two consecutive images of the SPLAL flight.

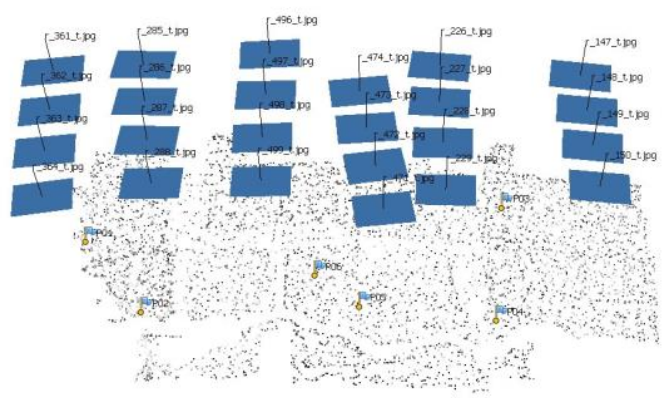

Figure 4 - Graphic representation of the oriented images, the sparse point cloud and the GCPs.

\subsection{Accuracy assessment over the DSM and orthomosaic}

The following step is, as in the UAV workflow, the generation of a DSM and an orthoimage mosaic. Over these two products we can analyze the global accuracy of the exterior orientation obtained. The orthoimage can be overlaid with other georeferenced images, from present time, or vector data, in order to check the planimetric accuracy. Vertical accuracy can be checked by comparing heights of the DSM and check points.

\subsection{Improvement of the GCP search}

The easiest way to collect GCPs for the image orientation is from orthoimages of the present time. Due to the significant changes that can occur, especially in areas around cities, finding GCPs in old photos may be very difficult, especially if the user does not know the area. A strategy was followed which facilitates this task.

For most of the available photos an approximate position of the image centre is known, in a similar way to what happens with UAV images. This allows for the creation of an orthoimage with an approximate georeferencing, without any GCPs. Although it may have large errors (100 meters or so) it can be overlaid with present time georeferenced imagery, in order to start the identification of common points to be used as GCPs. The orientation process is then iterated, now with the collected GCPs.

\section{APPLICATION TO SPLAL AND USAF PHOTOS}

The methodology was applied to several blocks of the available images, some with up to 40 photos. Results for two cases are described below, one with photos of the city of Porto from 1947 and another from a rural mountainous area, with USAF photos from 1958.

\subsection{Images from the city of Porto (SPLAL, 1947)}

A total of 24 photos were available from the SPLAL flights of 1947 , covering all the city of Porto. The image alignment was done with a total of 6581 conjugate points. GCPs were collected from present time orthophotos (planimetric coordinates) and 1:1000 scale topographic maps (heights), provided by the local authority. Heights within the area range from sea level to 150 meters. Although the area is familiar to the author, collection of good quality GCPs, at ground level, was very difficult, due to changes occurred in more than 60 years. For a total of 8 points, a root mean square errors smaller than 1 meter was obtained in .

The auto-calibration gave a new value for the focal distance of $200.9 \mathrm{~mm}$, instead of the nominal 204.4 marked on the photos. Tests were made considering radial or other distortion parameters but the results did not improve in terms of residuals. The resulting orthoimages (with or without distortion parameters) did not suffer significant changes, so it was concluded that no significant distortions existed.

Figure 5 shows the orthomosaic composed with the 24 photos. In order to assess the planimetric accuracy, check points should be used, but that was not done due to the difficulty in finding trustable points for this purpose. It was preferred to do a qualitative analysis by overlaying vector data, such as street axis, as shown in figure 6 . The agreement of the vector data with the existing streets of the time was always very good throughout the image, letting us conclude that the results of the orientation and camera calibration were acceptable.

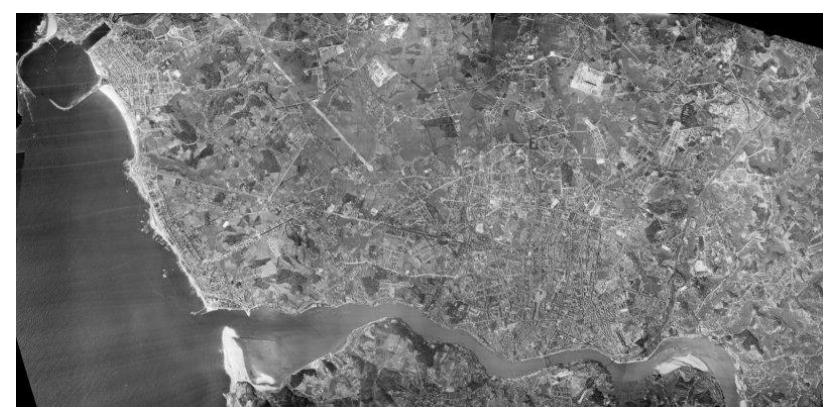

Figure 5 - Mosaic of 24 orthorectified photos of the city of Porto (SPLAL flight of 1947).

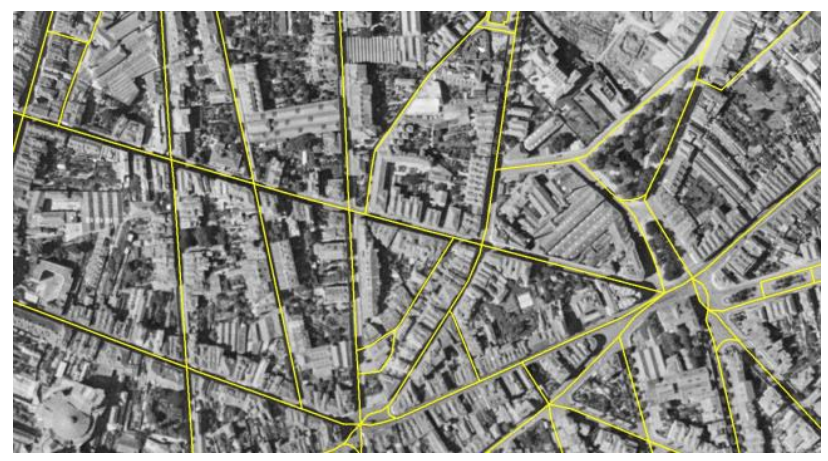

Figure 6 - Vector data overlaid on the orthophoto. Extension of the area; $750 \mathrm{~m}$ by $400 \mathrm{~m}$. 


\subsection{Photos from rural mountainous area}

Another set of 8 images of the USAF58 flight, in the region of Montalegre, a mointainous rural area in north Portugal, were also processed. Since the area was not familiar to the author, initial identification of GCPs in present time orthos was very difficult. The strategy of building an initial mosaic, with approximate georeferencing coming from the projection centers was very useful. After that, a total of 14 points were easily obtained, mainly in boundaries between fields, which frequently keep unchanged. Heights were obtained on topographic maps.

Figure 7 shows a sample of the orthomosaic (900 m by $500 \mathrm{~m}$ ). The Open Street Maps (OSM) layer was overlaid for quality assessment of the mosaic, which was in general good. Focal distance of the camera was changed from nominal value 152.42 $\mathrm{mm}$ to $152.70 \mathrm{~mm}$. The effects over the resulting orthophoto of considering or not the radial distortion parameters was negligible.

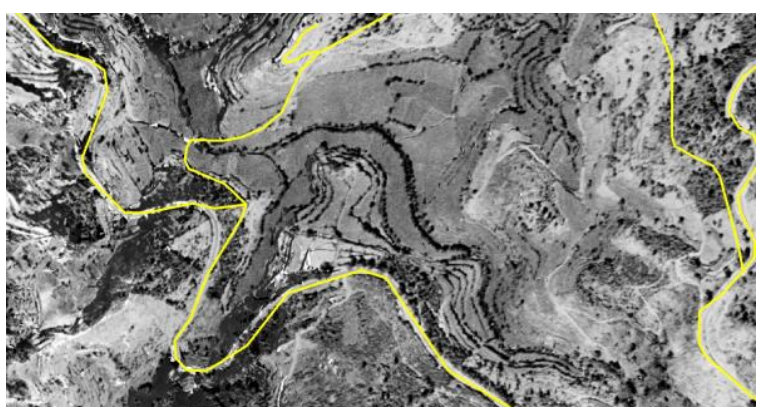

Figure 7 - Portion ( $900 \mathrm{~m}$ by $500 \mathrm{~m}$ ) of the USAF58 orthophoto in a mountainous area, with OSM vector layer.

Figure 8 shows a sample of the extracted DSM in the mountainous area. In order to assess its vertical accuracy, spot heights from topographic maps of scale 1:10,000 of the area were used as check points. Height errors were calculated for a total of 1183 points. Table 1 contains the statistics of these errors.

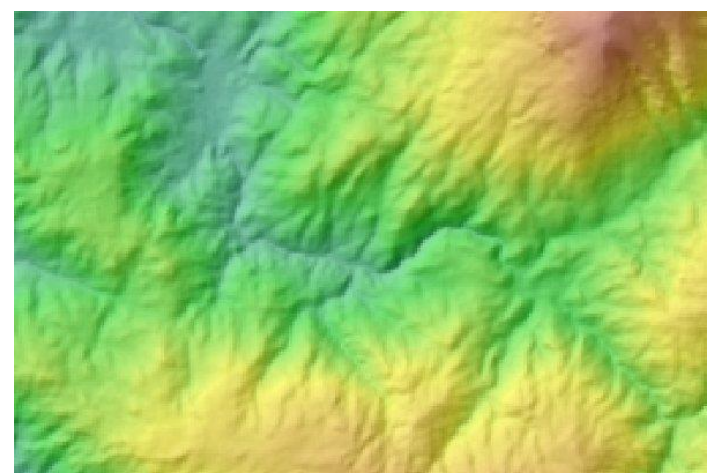

Figure 8 - Sample of the extracted DSM ( $5 \mathrm{~km}$ by $3 \mathrm{~km})$.

Table 1 - Statistics of the height errors found in 1183 altimetric check points

\begin{tabular}{|c|c|}
\hline & $\Delta \mathrm{H}(\mathrm{m})$ \\
\hline Minimum & -25.0 \\
Maximum & 22.7 \\
Mean & 1.2 \\
RMSE & 4.7 \\
\hline
\end{tabular}

\section{CONCLUSIONS}

The method proposed aims at providing orthomosaics of archived aerial photographs. It comprises the determination of exterior image orientation and camera auto-calibration, in an essentially automatic manner. Ground control is obtained from present time data. The method overcomes the lack of information of detailed camera characteristics, such as rigorous focal distance, or fiducial mark coordinates.

Tests were made with images of old photographs of Portugal. It proved to be applicable to process very large archives of aerial photos, in acceptable time and with reduced costs.

\section{REFERENCES}

Abdel-Aziz Y. I, Karara H.M. (1971). Direct Linear Transformation from Comparator Coordinates into Object Space Coordinates. Proceedings of the ASP Symposium on Close-Range Photogrammtery, Falls Church, VA, pp. 1-18., 1971.

Abdel-Wahab, M., Wenzel, K., \& Fritsch, D. (2012) Efficient Reconstruction of Large Unordered Image Datasets for High Accuracy Photogrammetric Applications, ISPRS Ann. Photogramm. Remote Sens. Spatial Inf. Sci., I-3, 1-6,

Agisoft, 2015. Agisoft documentation: http://www.agisoft.com /pdf/photoscan-pro_1_2_en.pdf (Visited in January 2014).

Alhaddad, B., Cladera, J.R., Burns, M.C. (2009). Monitoring Urban Sprawl from historical aerial photographs and satellite imagery using Texture analysis and mathematical morphology approaches. Proceedings of "Engineering of Reconfigurable Systems and Algorithms - ERSA'09", July 13-16, 2009. Nevada, USA.

Deseilligny, M.P., Clery, I. 2011. Apero, an open source bundle adjustment software for automatic calibration and orientation of set of images. In: Proceedings of ISPRS International Workshop on 3D Virtual Reconstruction and Visualisation of Complex Architectures, Trento, Italy, 2-4 March, 2011, Trento, Italy, pp. 269-276.

Lowe, D. 2004: Distinctive Image Features from Scale-Invariant Keypoints. International Journal of Computer Vision. Vol. 60, No. 2, pp. 91-110.

Ma, R., Buchwald, A. (2012). Orthorectify Historical Aerial Photographs Using DLT. Proceedings of the ASPRS 2012 Annual Conference, Sacramento, California. March 19-23, 2012.

Redweik, P., Roque, D., Marques, A., Matildes, R., Marques, F. (2010). Photogrammetric Engineering \& Remote Sensing, Vol. 76, No. 9, September 2010, pp. 1007-1018.

\section{ACKNOWLEDGEMENTS}

Images were provided by the "Instituto Geográfico do Exército" (IGeoE).

Data for the city of Porto was provided by the geographic information division of the Porto City Hall.

Global orthoimages of Portugal are provided by "Direcção Geral do Território". 